\title{
Confinement in 4D: An Attempt at Classical Understanding
}

\author{
Ibrahim Burak Ilhan ${ }^{1}$ (D) and Alex Kovner ${ }^{2, *(D)}$ \\ 1 Department of Physics, METU, Ankara 06800, Turkey; burakilhan@gmail.com \\ 2 Physics Department, University of Connecticut, 2152 Hillside Road, Storrs, CT 06269-3046, USA \\ * Correspondence: alexander.kovner@uconn.edu
}

check for

updates

Citation: Ilhan, I.B.; Kovner, A. Confinement in 4D: An Attempt at Classical Understanding. Universe 2021, 7, 291. https://doi.org/ 10.3390/universe7080291

Academic Editor: Dmitry Antonov

Received: 8 July 2021

Accepted: 4 August 2021

Published: 7 August 2021

Publisher's Note: MDPI stays neutral with regard to jurisdictional claims in published maps and institutional affiliations.

Copyright: (C) 2021 by the authors. Licensee MDPI, Basel, Switzerland. This article is an open access article distributed under the terms and conditions of the Creative Commons Attribution (CC BY) license (https:// creativecommons.org/licenses/by/ $4.0 /)$.

\begin{abstract}
In this review, we revisit our approach to constructing an effective theory for Abelian and Non-Abelian gauge theories in 4D. Our goal is to have an effective theory that provides a simple classical picture of the main qualitatively important features of these theories. We set out to ensure the presence of the massless photons-Goldstone bosons in Abelian theory and their disappearance in the Non-Abelian case-accompanied by the formation of confining strings between charged states. Our formulation avoids using vector fields and instead operates with the basic degrees of freedom that are the scalar fields of a nonlinear $\sigma$-model. The Mark 1 model we study turns out to have a large global symmetry group-the 2D diffeomorphism invariance in the Abelian limit, which is isomorphic to the group of all canonical transformations in the classical two dimensional phase space. This symmetry is not present in QED, and we eliminate it by "gauging" this infinite dimensional global group. Introducing additional modifications to the model (Mark 2), we are able to prove that the "Abelian" version is equivalent to the theory of a free photon. Achieving the desired property in the "Non-Abelian" regime turns out to be tricky. We are able to introduce a perturbation that leads to the formation of confining strings in our Mark 1 model. These strings have somewhat unusual properties, in that their profile does not decay exponentially away from the center of the string. In addition, the perturbation explicitly breaks the diffeomorphism invariance. Preserving this invariance in the gauged model as well as achieving confining strings in Mark 2 model remains an open question.
\end{abstract}

Keywords: confinement; higher order theories; gauge theory; effective field theory; magnetic flux symmetry

\section{Introduction}

Understanding confinement in Non-Abelian gauge theories is a long standing theoretical problem. There is very little doubt that QCD is confining. One has very strong indications of that from lattice gauge theory as well as from a variety of theoretical considerations. Nevertheless, a satisfactory simple understanding of the confinement phenomenon in $3+1$ dimensional theories is still missing. By such an understanding, we mean a simple qualitative picture that relies on universal concepts.

In $2+1$ dimensions, such a picture does exist. In this low dimensionality, one is able to directly relate confinement with a universal phenomenon of spontaneous symmetry breaking. The symmetry in question is a discrete symmetry generated by the magnetic flux [1-3]. The equivalence between confinement and a spontaneous breaking of magnetic symmetry provides a simple classical picture of the formation of a confining string.

There is an additional feature of gauge theories in $2+1$ dimensions that very much facilitates their qualitative understanding. Namely, the effective description of confining Non-Abelian gauge theories and Abelian nonconfining differs only by simple magnetic symmetry breaking deformation. The magnetic symmetry in the Abelian case is a continuous $U(1)$ group but is a discrete group $Z_{N}$ in $S U(N)$ gauge theories (without fundamental matter). This reduction of symmetry is affected in the effective Lagrangian by the presence of a simple deformation. The presence of this deformation, together with the spontaneous 
breaking of the discreet $Z_{N}$ group, unambiguously ensures an area law for Wilson loops and thereby a confining potential at long distances [2-4].

This review is devoted to a recent work that aims at constructing an analogous effective theory description in $3+1$ dimensions. The goal here is to "guess" an effective description that would display features similar to the $2+1$ dimensional case $[5,6]$. We design a model that embodies features of the transition between the Abelian and Non-Abelian regimes, similar to $2+1$ dimensions. Although it is not derived from QCD per se and therefore is not a bona fide QCD effective theory, amusingly, it does have some properties that have appeared before in the QCD context. In particular, the model has clear similarities with the Faddeev-Niemi model, which has been proposed as an effective theory of glueballs [7-11]. We note, however, that our perspective here is completely different, and we are not concentrating on the interpretation of knots as glueballs [7-11].

Prior to introducing our effective model, we will give a short recap of the confining physics in $2+1$ dimensional gauge theories. Consider the simplest Abelian gauge theoryQED with scalar Higgs fields. In addition to electric charge, it has a continuous magnetic global symmetry. The generator of this $U_{\mu}(1)$ group is the total magnetic flux through $2 \mathrm{D}, \Phi=\int d^{2} x B(x)$. As any proper global symmetry, $U_{\mu}(1)$ has an order parameter. In the present case, this is a complex field $V$, whose physical meaning is a field associated with creation and annihilation of point-like magnetic vortices. In the Coulomb phase, its expectation value does not vanish, $\langle V\rangle=v \neq 0$, and thus, the magnetic symmetry is spontaneously broken. One can easily write down an effective low energy theory that fits this simple symmetry breaking pattern and describes the low energy dynamics. The relevant model is defined by the Lagrangian

$$
L=-\partial^{\mu} V \partial_{\mu} V^{*}-\lambda\left(V^{*} V-\frac{e^{2}}{8 \pi}\right)^{2}
$$

The phase of the field $V$ appears in Equation (1) as a Goldstone boson associated with the spontaneous breaking of $U_{\mu}(1)$. This is nothing but the massless photon of QED. Interestingly, although the electric charge did not figure prominently in constructing Equation (1), it is indeed present in this description in the shape of the topological chargethe winding number of the field $V$

$$
J_{\mu}=\frac{1}{e} \epsilon_{\mu \nu \lambda} \partial_{\nu} V^{*} \partial_{\lambda} V
$$

A charged state of QED in the low energy description appears as a topological soliton of $V: V(x)=v e^{i \theta(x)}$, with $\theta=\tan ^{-1} y / x$. This description is frequently called a "dual" description as the basic fields used here are dual of the fields in the original QED Lagrangian, but a more physical view is that the Lagrangian Equation (1) is merely an effective low-energy long-distance Lagrangian of QED with scalar fields.

Equation (1) is a good starting point for understanding the confinement in NonAbelian gauge theories. Recall that in $2+1$ dimensions, confining theories have a weakly coupled regime. For example, the $S U(N)$ Higgs model at weak coupling is confining in the weakly coupled case. The appropriate low energy description for this theory is almost identical to Equation (1), with one important difference, i.e., an additional perturbation that breaks the magnetic $U_{\mu}(1)$ symmetry down to $Z_{N}$

$$
L=-\partial^{\mu} V \partial_{\mu} V^{*}-\lambda\left(V^{*} V-\frac{e^{2}}{8 \pi}\right)^{2}+\mu\left(V^{N}+V^{* N}\right)
$$

The presence of this additional potential has the effect of reducing the number of degenerate vacua of the Abelian theory (which is infinite) to a finite number of states connected by the $Z_{N}$ symmetry transformations. The effect of this reduction on the energy of a charged state is profound. A rotationally invariant "hedgehog" configuration now has an infinite energy proportional to the volume of the system. The lowest state with the nonvanishing winding number ("color charge") is not rotationally invariant anymore but instead has the winding concentrated within a quasi one-dimensional "flux tube" [2,3]. Its 
energy is proportional to the length of the flux tube and thus leads to linear confinement of charges.

The identification of electric (or "color") charges with topological defects in the effective theory is intuitively very appealing. Topological defects naturally have long range interactions due to their inherently nonlocal nature, which, in conjunction with spontaneous symmetry breaking, leads directly to linear confinement. Additionally, the identification of photons with Goldstone bosons in the Abelian limit furnishes a natural universal explanation for the fact that the photon is strictly massless.

The question arises if a similar description can be achieved in $3+1$ dimensions. One would like this description to encompass the Goldstone boson nature of photons in QED as well as an interpretation of confinement in terms of topological charges in Non-Abelian theories. Of course, life in $3+1$ dimensions is not at all that simple. First off, photons now are vector particles and thus, their interpretation as Goldstone bosons in the standard sense is questionable. Even if one successfully argues in favor of this, identification of the relevant conserved current that breaks spontaneously is far from straightforward. Clearly, this current has to be intimately tied with the dual field strength $\tilde{F}_{\mu v}$ since the photon is a spin one particle [12]. The identification of photons as Goldstone bosons of this higher form symmetry was achieved a while ago in [12] and was revived recently in [13]. The dual field strength, however, is an object of a very different nature than an ordinary vector current since no local order parameter that carries its charge can be defined even in principle. One might hope that a more conventional picture of symmetry breaking coexists with the "generalized symmetry" explanation, and it would be useful to clarify this. Another significant stumbling block is that we do not know of weakly coupled confining theories in $3+1$ dimensions. QCD is certainly strongly interacting while a classical effective description of the type described before is directly applicable only for a weakly interacting theory.

These are hard problems to solve, much too hard for the present modest attempt. Instead of addressing them head on here, we will largely ignore them and instead will simply try to construct a model that encompasses the basic properties described above:

1. The degrees of freedom of the model must be scalar fields, and no fundamental gauge fields should be involved.

2. A well-defined "Abelian regime" should be clearly definable. In this regime, two massless degrees of freedom should exist. These massless particles should be Goldstone bosons and as far as possible must have the properties of photons.

3. The Abelian regime should allow for the existence of classical topological solitons associated with the nontrivial topology of the manifold of vacua. These solitons represent electrically charged particles. More precisely, we would like the topological charge of the solitons to be associated with the mapping of the spatial infinity onto the manifold of vacua and thus be identified with $\Pi_{2}(M)$. Charged particles in QED are excitations of finite energy, and thus, the classical energy of the solitons must be infrared finite, and more precisely, the energy density of a soliton solution away from the position of the soliton must decrease as the fourth power of the distance. This is nontrivial in $3+1$ dimensions since our model has no gauge fields, while scalar fields that contribute to $\Pi_{2}$ have to be long range.

4. A "Non-Abelian regime" of the model is achieved by adding a perturbation that breaks explicitly the symmetry, which leads to the appearance of Goldstone bosons in the Abelian case. The Goldstone bosons now disappear from the spectrum or, more precisely, acquire a finite mass. In addition, in this Non-Abelian situation, the solitons do not disappear on small spatial scales, but they must become confined by a linear potential. The linear potential should arise due to the formation of a "string" or "flux tube" with finite linear energy density between the solitons.

In the first part of this review, we discuss a model (Mark 1) that exhibits all the above features. The Abelian version of the model has, in fact, been studied some years ago from a completely different perspective in [14] as a possible variation of Maxwell's theory. 
The properties of this model turn out to be a little unusual. In particular, as we will see, requiring the energy of a soliton in the Abelian regime to be finite puts a very strong restriction on possible forms of the kinetic term for the scalar fields. This noncanonical kinetic term results in rather unusual properties of confining strings once the symmetry breaking perturbation is introduced. In particular, the "Non-Abelian string" is forced into having an infinite number of zero modes. This infinite degeneracy can be avoided, but the price one has to pay is adding another perturbation that does not have a natural place in the paradigm described above.

Although the model has many nice features, it does not perfectly emulate many properties of gauge theories. Most importantly, in the Abelian regime, it has more classical solutions than allowed by the structure of Abelian gauge theories; in particular, some of them carry nonvanishing magnetic charge density. Thus, the field playing the role of the dual field strength tensor is not conserved in Mark1. A related problem is that we are not able to find classical solutions that can represent arbitrary multiphoton states. Although solutions of equations of motion that behave as single photons can be constructed, we show that there are no solutions that correspond to a two-photon state with arbitrary photon polarization.

This is partly due to the fact that the global symmetry group of the model turns out to be much larger than naively anticipated. The global symmetry group turns out to be isomorphic to diffeomorphism symmetry in two dimensions. These diffeomorphism transformations act nontrivially on the Hilbert space even though the fields that we identify with the electric and magnetic fields of QED are invariant under their action. QED does not possess such a large global symmetry.

We then discuss an approach devised to eliminate this global symmetry, which amounts to "gauging" it. The framework we work in is very different from the usual gauge theories, where "gauging" amounts to eliminating a set of local degrees of freedom. In our case, gauging applies only to global group of transformations and therefore does not change the number of local degrees of freedom.

Unfortunately, although we are able to eliminate the global diffeomorphisms from the model, it turns out not to be enough to bring it into full conformity with QED. We, therefore, take a different track and discuss a modification (Mark 2), which circumvents this obstacle. We show that the the model Mark 2, which shares many features with Mark 1, is indeed equivalent to the theory of a free Maxwell field in $3+1$ dimensions. However, even though we are able to reproduce the Abelian limit, introducing a reasonable Non-Abelian perturbation turns out to be quite tricky. We make some comments on how this can be achieved, but the implementation is left for the future.

\section{The Abelian Model: Mark 1}

\subsection{The Field Space and the Lagrangian}

As explained in the introduction, our aim is to devise a model containing scalar fields only with two massless degrees of freedom, which allows for finite energy solitons. We, thus, zero in on a theory of two scalar fields. In order to have a chance to get Goldstone bosons, we endow it with $S U(2)$ symmetry. The simplest option open for us is an $O(3)$ nonlinear $\sigma$-model.

$$
\phi^{a}, a=1,2,3 ; \quad \phi^{2}=1
$$

This moduli space allows for a nontrivial topology $\Pi_{2}\left(S^{2}\right)$. We will identify the corresponding topological charge with the electric charge of QED

$$
Q=\frac{e}{4 \pi^{2}} \int d^{3} x \epsilon_{a b c} \epsilon_{i j k} \partial^{i} \phi^{a} \partial_{j} \phi^{b} \partial_{k} \phi^{c}
$$

This identification when extended to current density suggests the following representation of the electric current and, by extension, of the electromagnetic field in the effective description:

$$
J^{\mu}=\frac{e}{4 \pi^{2}} \epsilon_{a b c} \epsilon^{\mu \nu \lambda \sigma} \partial_{\nu} \phi^{a} \partial_{\lambda} \phi^{b} \partial_{\sigma} \phi^{c}
$$




$$
F^{\mu v}=\epsilon^{a b c} \epsilon^{\mu v \lambda \sigma} \phi^{a} \partial_{\lambda} \phi^{b} \partial_{\sigma} \phi^{c}
$$

Our initial challenge is the following potential problem. A standard two derivative kinetic term would lead to infrared divergent energy of a soliton carrying a nonvanishing topological charge of Equation (5). Consider the simplest topologically nontrivial field configuration:

$$
\phi_{h}^{a}(x)=\frac{r^{a}}{|r|} f(|r|) ; \quad f(|r|) \rightarrow_{r \rightarrow \infty} 1
$$

The derivatives of the field decrease as the first power of the distance away from the soliton core, and therefore, the standard two derivative kinetic term gives energy, which diverges linearly in the infrared. The only way to cure this divergence is to not allow a two derivative kinetic term but instead consider a kinetic term with more than two derivatives.

Some reflection shows that there is a unique four derivative term that would do the job, which is also the most natural choice from another point of view. Since our goal is to approach the QED as close as possible, the natural choice for the kinetic term is the square of the field strength tensor in Equation (7). When written in terms of the scalar field $\phi^{a}$, this is just the well-known Skyrme term.

We, thus, consider a somewhat unusual nonlinear $\sigma$-model, which is defined by the Lagrangian:

$$
L=\frac{1}{16 e^{2}} F^{\mu v} F_{\mu \nu}+\lambda\left(\phi^{2}-1\right)^{2}
$$

with $F^{\mu v}$ defined in Equation (7).

At first sight, it may seem strange that the sign of the $F^{2}$ term in the Lagrangian Equation (9) is positive, while in QED, the same term enters with the negative sign. The sign in Equation (9) is determined by the requirement of positive definiteness of the Hamiltonian and is thus nonnegotiable. However, the reversal of the sign of the kinetic term is a staple of dual models. The $2+1$ dimensional models described in the introduction exhibit the same feature. In the Lagrangian of the effective theory, the kinetic term is the standard $\left|\partial_{\mu} V\right|^{2}$, while in QED, it is of course $-\tilde{F}_{\mu}^{2}$. With the identification of $V^{*} \partial_{\mu} V \propto \tilde{F}_{\mu}$, the signs of the two kinetic terms are again opposite. The reason for this inversion is that, while in QED, the electric field is proportional to the time derivative of the basic field (in this case, $A_{\mu}$ ). In the effective dual description, it is the magnetic field that contains the time derivative of the vertex field $V$. Thus, in order for the Hamiltonian of the two models to be the same, the kinetic terms in the respective Lagrangians must have opposite signs.

In Equation (9), we have introduced a coupling $\lambda$. The role of this coupling is easy to understand. In the strong coupling limit $\lambda \rightarrow \infty$, the isovector $\phi$ is forced to have a unit length, and we are back to Equation (4). In this limit, the field strength is trivially conserved

$$
\partial_{v} F^{\mu v}=0
$$

which means that electric current vanishes. Therefore, the strong coupling limit should correspond to the pure Maxwell theory: the energy of the soliton Equation (8) at strong coupling diverges linearly in the ultraviolet. At finite $\lambda$, the radial component of the field $\phi^{a}$ can vary in space and vanishes in the soliton core. This eliminates the UV divergence of the energy, and the soliton has a finite mass. The finite value $\lambda$, therefore, corresponds to an Abelian theory with charged matter of finite mass. We again stress that the energy of the soliton is also finite in the infrared, thanks to our choice of the four derivative action. For the hedgehog configuration in Equation (8), the "electric field" decreases as $E_{i}(x) \propto \frac{\hat{r}_{i}}{r^{2}}$, and the energy density away from the soliton core decreases as $1 / r^{4}$. This is the same as the behavior of the Coulomb energy of a static electric charge in the electrodynamics.

Interestingly, the same model was discussed a while ago in [14] with an entirely different motivation. 


\subsection{The Equations of Motion}

Given the Lagrangian, we can now write down equations of motion for our effective theory. We work in the strong coupling limit and define two independent degrees of freedom as

$$
\phi_{3}=z, \quad \psi=\phi_{1}+i \phi_{2}=\sqrt{1-z^{2}} e^{i \chi}
$$

With this definition, we have

$$
F^{\mu v}=\epsilon^{\mu \nu \alpha \beta} \epsilon_{a b c} \phi^{a} \partial_{\alpha} \phi^{b} \partial_{\beta} \phi^{c}=-2 \epsilon^{\mu v \alpha \beta} \partial_{\alpha} z \partial_{\beta} \chi
$$

The Lagrangian becomes

$$
L=\frac{1}{4 e^{2}}\left(\partial_{\mu} z \partial_{\nu} \chi-\partial_{\mu} \chi \partial_{\nu} z\right)^{2}
$$

and the equations of motion are

$$
\begin{aligned}
& \partial^{\mu}\left[\frac{1}{e^{2}} \partial^{\nu} \chi\left(\partial_{\mu} z \partial_{\nu} \chi-\partial_{\nu} z \partial_{\mu} \chi\right)\right]=0 \\
& \partial^{\mu}\left[\frac{1}{e^{2}} \partial^{\nu} z\left(\partial_{\mu} z \partial_{\nu} \chi-\partial_{\nu} z \partial_{\mu} \chi\right)\right]=0
\end{aligned}
$$

Interestingly, these equations can be written as

$$
\frac{1}{e^{2}} \partial_{\nu} G(z, \chi) \partial_{\mu}\left(\partial_{\mu} z \partial_{\nu} \chi-\partial_{\nu} z \partial_{\mu} \chi\right)=\frac{1}{e^{2}} \partial_{\nu}\left[G(z, \chi) \partial_{\mu}\left(\partial_{\mu} z \partial_{\nu} \chi-\partial_{\nu} z \partial_{\mu} \chi\right)\right]=0
$$

with $G(z, \chi)$ being an arbitrary function of two variables. These can be thought of as an infinite number of conservation equations, where the conserved current corresponding to a given function $G(z, \chi)$ is defined as

$$
J_{v}^{G}=G(z, \chi) \partial^{\mu}\left(\partial_{\mu} z \partial_{\nu} \chi-\partial_{\nu} z \partial_{\mu} \chi\right)
$$

We will see later that the existence of an infinite number of conserved currents is a very important feature.

\subsection{The Symmetries of the Model and Correspondence to Electrodynamics}

Given that we have identified and infinite number of conserved currents from Equation (16), we see that the choice of the Skyrme term as the kinetic term in the Lagrangian allows a very large global symmetry group of the model. The global symmetry group of Equation (9) is not just the $S O(3)$ group that we required from the outset but is isomorphic to the group of diffeomorphisms in two dimensions.

This is easy to understand. The field strength in Equation (7) is related to an infinitesimal area element on a configuration space. A given field configuration $\phi^{a}(x)$ defines a map from space-time to a sphere $S^{2}$. Consider a given component, such as the field strength tensor, say $F_{12}$ at some point $x$. To express it in terms of $\phi$, we consider three infinitesimally close points $A \equiv x^{\mu}, B \equiv x^{\mu}+\delta^{\mu 1} a$, and $C \equiv x^{\mu}+\delta^{\mu 2} a$. These three points in space-time map into three infinitesimally close points on the sphere $\phi^{a}(A), \phi^{a}(B), \phi^{a}(C)$. The field strength $F_{12}$ is proportional (up to the factor $a^{-2}$ ) to the area of the infinitesimal triangle on $S^{2}$ defined by these three points. Since our Lagrangian depends only on $F^{\mu v}$, clearly an arbitrary area preserving reparametrization of the sphere leaves our action unchanged.

The original $S O(3)$ global symmetry is only a small subgroup of the area preserving diffeomorphisms of $S^{2}$ - the group we will denote $\operatorname{Sdiff}\left(S^{2}\right)$ [15]. As an aside, we note that this group is also isomorphic to the group of canonical transformations on a classical two-dimensional phase space. The infinitesimal $\operatorname{Sdiff}\left(S^{2}\right)$ transformation when acting on $z$ and $\chi$ is written as

$$
z \rightarrow z+\frac{\partial G}{\partial \chi} ; \chi \rightarrow \chi-\frac{\partial G}{\partial z}
$$


The Noether currents are those given by Equation (16), and the equations of motion are equivalent to conservation equations of these currents.

An intriguing point is that the symmetry Equation (17) is reminiscent of the world sheet diffeomorphism invariance of the Nambu-Gotto string. Indeed, if the fields $z$ and $\chi$ are thought as the world sheet string coordinates, the world sheet diffeomorphism invariance is precisely Equation (17). The model discussed here is not motivated by a string theory and a priori has nothing to do with a string theory. Nevertheless, the similarities may run deeper than just a coincidence since the fundamental "order parameters" of the magnetic symmetry in $3+1$ dimensions are indeed magnetic vortex strings [12]. The $S^{2}$ topology of the world sheet would then ask for closed string loops. The analogy is indeed intriguing and would be worthwhile pursuing further, but since this is not the goal of our exploratory efforts here, we will return to the field theoretical approach in the rest of this review.

The fact that the global symmetry group of the model is so large means that the moduli space (space of all vacuum configurations) is much larger than $S^{2}$, which corresponds to a symmetry breaking pattern $S O(3) \rightarrow S O(2)$. Consider an arbitrary field configuration $\phi^{a}(x)$ that maps the configuration space into any one-dimensional curve on $S^{2}$. Such a configuration has a vanishing action and therefore is a classical vacuum. The full moduli space is, therefore, the union of maps $\phi^{a}(x)$ that map $R^{4}$ to $L$, where $L$ is an arbitrary point or a one-dimensional curve on $S^{2}$.

Still the important question for us is whether the topology of this moduli space is right to support classically quantized topological charge. Indeed, from its definition, it is clear that the topological charge $Q$ is quantized on any smooth classical configuration of fields $\phi(x)$. The catch is that there are many more degenerate soliton configurations than just the rotationally invariant hedgehog of Equation (8). Any $\operatorname{Sdiff}\left(S^{2}\right)$ transformation with an arbitrary (regular) function $G$ of Equation (17) applied to Equation (8) generates a configuration $\phi_{h}^{a G}(x)$, which carries the same charge $Q$ as $\phi_{h}^{a}(x)$ and is degenerate with it in energy. However, although these are different field configurations, they all have the same electric field $E_{i}=\epsilon_{i j k} \epsilon^{a b c} \phi^{a} \partial_{j} \phi^{b} \partial_{k} \phi^{c}$ since the field strength is invariant under the action of $\operatorname{Siff}\left(S^{2}\right)$. Thus, if one is physically only allowed to measure electromagnetic fields, all these solitons look identical.

\subsection{The Photon States-a.k.a. Plane Waves}

We have constructed the Lagrangian Equation (9) so that it has the maximal similarity to QED when written in terms of the putative electromagnetic fields. This does not yet ensure that the content of the theory is the same as that of electrodynamics. We do know that the field strength $F^{\mu v}$ identified in Equation (7) satisfies half of Maxwell's equationsthe Coulomb law and the evolution equations for electric field. The other half of Maxwell's equations (dynamics of magnetic fields) have to follow from the equations of motions of our model. Indeed, there is clear similarity between Equation (15) and the Maxwell's equations. Equation (15) can be rewritten in terms of $F^{\mu v}$ as

$$
\left[\partial_{\nu} G(z, \chi)\right] \partial_{\mu} \tilde{F}^{\mu \nu}=0
$$

This ensures that for any configuration of the fields $z$, the $\chi$ that satisfies $\partial_{\mu} \tilde{F}^{\mu \nu}=0$ also satisfies the equations of motion of our model. However, the converse is not assured. In Appendix A, we give an example of a solution of Equation (15) that does not satisfy the equations of motion of electrodynamics.

Thus, there is no full equivalence between the model Equation (9) and electrodynamics. Nevertheless, we can ask to what extent the spectrum of solutions of Equation (9) contains basic excitations of QED. The excitations of particular interest in the present context are of course the photons. (Although we are dealing with a classical theory and not a quantum theory, we will, with a slight abuse of language, refer to plane wave configurations of $F^{\mu v}$ with light-like momentum as photons). 
We aim now to show that free wave excitations are indeed solutions of Equation (15). Consider the following configuration

$$
\chi(x)=A \epsilon^{\mu} x_{\mu} ; \quad z(x)=\sin k^{\mu} x_{\mu}
$$

where the vector $\epsilon^{\mu}$ is normalized as $\epsilon^{\mu} \epsilon_{\mu}=-1$. Calculating the field strength, we find

$$
\tilde{F}^{\mu v}=A\left(\epsilon^{\mu} k^{\nu}-\epsilon^{v} k^{\mu}\right) \cos k \cdot x
$$

Thus,

$$
\partial_{\mu} \tilde{F}^{\mu v}=-A\left[(\epsilon \cdot k) k^{v}-k^{2} \epsilon^{v}\right] \sin k \cdot x
$$

If the momentum vector is light-like and the polarization vector $\epsilon$ is perpendicular to $k$, this vanishes:

$$
k^{2}=0 ; \epsilon \cdot k=0
$$

For a fixed light-like momentum $k_{\mu}$, Equation (22) has three independent solutions for $\epsilon^{\mu}$, one of which is proportional to $k_{\mu}$. For $\epsilon_{\mu} \propto k_{\mu}$, the field strength tensor Equation (19) vanishes, and so, there are two independent polarization vectors $\epsilon_{\lambda^{\prime}}^{\mu}, \lambda=1,2$ that yield plane wave solutions for $F^{\mu \nu}$. We can always choose the polarization vectors so that their zeroth component vanishes $\epsilon_{\lambda}^{\mu}=\left(0, \epsilon_{\lambda}^{i}\right)$, just like in electrodynamics. The square of the overall amplitude of wave $A$ in the quantum case is proportional to the number of photons with a given momentum and a given polarization vector.

Note that the freedom in the choice of the independent polarization vectors is exactly the same as in electrodynamics

$$
\epsilon^{\mu} \rightarrow \epsilon^{\mu}+a k^{\mu}
$$

This change of polarization vector is generated by the transformation

$$
\chi \rightarrow \chi+a \arcsin z
$$

which is a particular element of the $\operatorname{Sdiff}\left(S^{2}\right)$ group from Equation (17). More generally, the field configuration in Equation (19) can be transformed by any element of $\operatorname{Sdiff}\left(S^{2}\right)$ without causing a change in $F^{\mu \nu}$.

The solution Equations (19)-(22) describe a state that in all respects is equivalent to the freely propagating photon, and we will refer to it as such. The solution Equation (19) suggests an intuitive interpretation for the properties of the photon states in terms of the effective theory. The momentum of the photon is simply the momentum associated with the variation of the third component of the isovector $\phi^{a}$, while the direction of the photon polarization vector is the direction of the spatial variation of the phase $\chi$.

We again note that the present formulation is easier to interpret in terms of quantities dual to those normally used in QED. One usually introduces the vector potential $A_{\mu}$ via $F^{\mu \nu}=\partial_{\mu} A_{v}-\partial_{\nu} A_{\mu}$, which potentiates the homogeneous Maxwell's equation $\partial_{\mu} \tilde{F}^{\mu \nu}=0$. However, in the absence of electric charges, one can alternatively potentiate the other half of Maxwell's equation by introducing the dual vector potential via $\tilde{F}^{\mu \nu}=\partial_{\mu} \tilde{A}_{\nu}-\partial_{\nu} \tilde{A}_{\mu}$. In the absence of charges, the dynamics of the dual vector potential $\tilde{A}_{\mu}$ is identical to that of $A_{\mu}$, and it can be expanded in exactly the same polarization basis as $A_{\mu}$. In this dual formulation, QED possesses a dual gauge symmetry $\tilde{A}_{\mu} \rightarrow \tilde{A}_{\mu}+\partial_{\mu} \lambda(x)$.

To make the correspondence to our model more obvious, we can introduce a "dual vector potential" by

$$
\tilde{A}_{\mu}=z \partial_{\mu} \chi
$$

As opposed to the field strength tensor itself, this object is not invariant under the $\operatorname{Sdiff}\left(S^{2}\right)$ group transformation from Equation (17):

$$
\tilde{A}_{\mu} \rightarrow \tilde{A}_{\mu}+\partial_{\mu}\left[G-z \frac{\partial G}{\partial z}\right]
$$


This is similar to the dual gauge transformation in electrodynamics with the gauge function $\lambda(x)=G-z \frac{\partial G}{\partial z}$.

The analogy of Equation (25) is suggestive, but one has to keep in mind that this is not at all an equivalence. First, the transformation Equation (26) is not a gauge transformation but rather the action of a global symmetry transformation of the Lagrangian on $\tilde{A}_{\mu}$ of Equation (25). More importantly, an arbitrary vector field cannot be expressed in terms of two scalars by a relation of the type Equation (25), even allowing for a possible gauge ambiguity. Thus, Equation (25) cannot be considered merely a convenient parametrization of the dual potential of electrodynamics. For this reason, the variation of the Lagrangian Equation (9) with respect to such a constrained vector potential does not lead to directly to homogeneous Maxwell's equations but instead to Equation (18).

We have thus determined that monochromatic plane wave $\tilde{F}_{\mu \nu}$ solves the equations of motion of our effective model. In QED, which is a linear theory of $F_{\mu v}$, the immediate consequence is that a superposition of such waves is a solution as well. However, Equation (18) is not linear in the basic field variables, and thus, a superposition of two such solutions is not assured to be a solution as well. Let us try to construct a "two photon state" by slightly extending the ansatz Equation (19).

$$
\chi=\lambda_{\mu} x_{\mu} ; \quad z=a \sin k^{\mu} x_{\mu}+b \sin p^{\mu} x_{\mu}
$$

with $k^{\mu}$ and $p^{\mu}$-both light-like vectors, $\lambda^{\mu} k_{\mu}=\lambda^{\mu} p_{\mu}=0$ and $\lambda^{\mu} \lambda_{\mu}=-1$. The latter two conditions can be satisfied by taking

$$
\lambda^{\mu}=\alpha\left[\epsilon^{\mu}-\frac{\epsilon \cdot k}{k \cdot p} p_{\mu}-\frac{\epsilon \cdot p}{k \cdot p} k_{\mu}\right]
$$

with an arbitrary vector $\epsilon^{\mu}$ and an appropriate normalization constant $\alpha$.

The dual field strength tensor is now:

$$
\tilde{F}_{\mu \nu}=a\left(k_{\mu} \epsilon_{v}^{k}-k_{\nu} \epsilon_{\mu}^{k}\right) \cos k \cdot x+b\left(p_{\mu} \epsilon_{v}^{p}-p_{v} \epsilon_{\mu}^{p}\right) \cos p \cdot x
$$

with

$$
\epsilon_{\mu}^{k}=\lambda_{\mu}-\frac{\lambda_{0}}{k_{0}} k_{\mu} ; \quad \epsilon_{\mu}^{p}=\lambda_{\mu}-\frac{\lambda_{0}}{p_{0}} p_{\mu} ;
$$

This looks like a bona fide two-photon state. However, our ansatz does not yield a generic two-photon state with arbitrary polarization vectors: both the polarization vectors $\epsilon^{k}$ and $\epsilon^{p}$ above have equal components in the perpendicular direction to the plane spanned by $p^{i} ; k^{i}$. Thus, we are one degree of freedom short and cannot construct a two-photon state with arbitrary polarizations of both photons. Although this might look merely like a limitation of our particular ansatz, we show in Appendix A that this problem is not restricted to the ansatz Equation (27) but is unfortunately a genuine limitation of our effective model.

\section{Going Non-Abelian: The "Confining String"}

Our main goal in this project is to have a model representation of the confinement phenomenon in Non-Abelian theories. We, therefore, take the same trek as in $2+1$ dimensions. Namely, we will add to the Lagrangian Equation (9) a simple perturbation that explicitly breaks the global symmetry of the model. This modification of low energy description is meant to get rid of the multiple vacuum structure inherent to spontaneous symmetry breaking and therefore eliminate massless excitations. For convenience, we will choose a potential that (classically) sets the vacuum expectation value of the field $z$ to unity.

With the above considerations, we are led to consider the Lagrangian

$$
L=\frac{1}{16 e^{2}} F^{\mu v} F_{\mu v}-\frac{2}{e^{2}} \Lambda^{2}(z-1)^{2}
$$


The potential we have added of course breaks the $S O(3)$ symmetry, but in addition, it is also not invariant under a general $\operatorname{Sdiff}\left(S^{2}\right)$ transformation. However, the $\operatorname{Sdiff}\left(S^{2}\right)$ is not broken completely but only up to the subgroup

$$
\chi \rightarrow \chi-\frac{d G(z)}{d z}
$$

We keep this in mind throughout the discussion of this section.

The equations of motion of the perturbed model are

$$
\begin{aligned}
& \partial^{\mu}\left[\frac{1}{e^{2}} \partial^{v} \chi\left(\partial_{\mu} z \partial_{\nu} \chi-\partial_{\nu} z \partial_{\mu} \chi\right)\right]=\frac{4}{e^{2}} \Lambda^{2}(z-1) \\
& \partial^{\mu}\left[\frac{1}{e^{2}} \partial^{v} z\left(\partial_{\mu} z \partial_{\nu} \chi-\partial_{\nu} z \partial_{\mu} \chi\right)\right]=0
\end{aligned}
$$

These equations do not have static topologically stable solutions of finite energy. However, one can still ask what is the energy of a configuration of a soliton and antisoliton separated far in space. As the answer to this question, we expect to find a (approximately) translationally invariant string-like configuration that connects the soliton and the antisoliton and produces a linear confining potential between the two. Consider a static field configuration translationally invariant in the third direction. The only components of $F_{\mu v}$ that do not vanish then are:

$$
F^{03}=2 \epsilon^{i j} \partial_{i} z \partial_{j} \chi
$$

Let us take the following ansatz, which preserves rotational symmetry in the $x_{1}-x_{2}$ plane:

$$
\chi(x)=\theta(x) ; \quad z(x)=z(r)
$$

Here, $r$ and $\theta$ are the polar coordinates in the $x_{1}, x_{2}$ plane. This ansatz parametrizes a configuration with a unit winding in the $x_{1}, x_{2}$ plane, which should be the case for a string connecting a soliton and an antisoliton. The soliton partner of the pair is located at a very large negative value of $x_{3}$. At even more negative $x_{3}$, the field must relax into the vacuum $\phi^{1}=\phi^{2}=0 ; z=1$. Therefore, the topological charge calculated on a surface enclosing the soliton (but not its antisoliton partner) should be given by the two dimensional topological charge - the winding number of the phase $\chi$ on any surface crossed by the string. The same argument applies for the antisoliton, which resides at large positive value of $x_{3}$. Our ansatz, therefore, describes a confining string connecting a widely separated soliton-antisoliton pair.

Interestingly, the equation of motion for the field $\chi$ is automatically satisfied for Equation (35). The only nontrivial equation is that for $z$ :

$$
4 z^{\prime \prime}=4 \Lambda^{2}(z-1)
$$

with $z^{\prime} \equiv \frac{d z}{d\left(r^{2}\right)}$

In order for the solution to have finite linear energy density, $z$ must satisfy the boundary conditions:

$$
z(0)=-1, z(\infty)=1
$$

The solution with these boundary conditions is

$$
z\left(r^{2}\right)=1-2 \exp \left\{-\Lambda r^{2}\right\}
$$

Some of the properties of this solution are intuitively appealing. It has a finite width governed by the only dimensional parameter $\Lambda$. Outside of this width, the fields relax to 
vacuum. Inside the string, the potential energy is finite, and thus, the string carries finite linear energy density. The string tension is found to be

$$
\sigma=8 \pi \frac{\Lambda}{e^{2}}
$$

One feature of the solution, however, is rather peculiar. Away from the string core, the fields do not approach the vacuum configuration exponentially but rather as a Gaussian in transverse distance. The string profile is, therefore, unusual as it has a very sharp boundary, outside of which the vacuum is reached very quickly. Such a behavior is unusual and, in fact, is not possible in a local field theory with a finite mass gap and a finite number of massive excitations. We can trace the origins of this behavior back to the non canonical kinetic term in Equation (9), which has four derivatives. For simple dimensional reasons, the kinetic energy for a rotationally invariant configuration is given by the second derivative with respect to $r^{2}$ rather than $r$, which results in a Gaussian rather than exponential decay of the solution fields.

\section{The $Z_{N}$ Preserving Perturbation}

The potential of Equation (31) breaks the $S O(3)$ as well as the $\operatorname{Sdiff}\left(S^{2}\right)$ symmetries but leaves an $O(2)$ subgroup of $S O(3)$ and a large subgroup $\operatorname{Sdiff}\left(S^{2}\right)$ (Equation (32)) unbroken. On the other hand, if we follow a direct analogy with $2+1$ dimensions, we expect the effective theory in the Non-Abelian regime to preserve only a $Z_{N}$ subgroup of $S O(3)$. We can easily implement such a perturbation in the effective theory. Let us modify the Lagrangian to

$L=\frac{1}{16 e^{2}} F^{2}-\frac{2}{e^{2}} \Lambda^{2}(z-1)^{2}\left[1-\mu\left(\psi^{N}+\psi^{\star N}\right)\right]=\frac{1}{16 e^{2}} F^{2}-\frac{2}{e^{2}} \Lambda^{2}(z-1)^{2}\left[1-2 \mu\left(1-z^{2}\right)^{N / 2} \cos N \chi\right]$

For large enough $\mu$, the additional perturbation shifts the lowest energy value away from $z=1$. For simplicity, we will only consider values

$$
\mu<\frac{1}{2}
$$

for which the vacuum configuration remains at $z=1$.

We will now study the effect of the additional perturbation on the structure of the "confining string".

Assuming a long string in the direction $x_{3}$, the energy per unit length can be written as

$$
E=\int d^{2} x \frac{1}{2 e^{2}}\left(\epsilon_{i j} \partial_{i} z \partial_{j} \chi\right)^{2}+\frac{2}{e^{2}} \Lambda^{2}(z-1)^{2}\left[1-2 \mu\left(1-z^{2}\right)^{N / 2} \cos N \chi\right]
$$

\subsection{Perturbative Solution}

For small values $\mu \ll 1$, we can find corrections to the string solution perturbatively. Let us take the following ansatz for the perturbative solution:

$$
z(r, \theta)=z(r) ; \quad \chi=\theta+\chi_{1}(r, \theta)=\theta+f(r) \sin N \theta
$$

where $z(r)$ is given by Equation (38). Although this is not the most general possible form of the perturbative correction, it nevertheless yields a solution to first order in $\mu$, as we now show.

The leading order perturbative equation for $f$ is

$$
\frac{1}{e^{2}} 8 N^{2}\left(z^{\prime}\right)^{2} f \sin N \theta=\frac{1}{e^{2}} N \mu \Lambda^{2}(z-1)^{2}\left(1-z^{2}\right)^{N / 2} \sin N \theta
$$

which is solved by

$$
f\left(r^{2}\right)=\frac{\mu}{N}\left[2 e^{-\Lambda r^{2}}\left(1-e^{-\Lambda r^{2}}\right)\right]^{N / 2}
$$


In principle, we have to consider also the minimization equation for $z(r)$. It reads

$$
\frac{1}{e^{2}} 8 N\left[2 z^{\prime \prime} f+z^{\prime} f^{\prime}\right]=\frac{1}{e^{2}} 4 \mu \Lambda\left[2(z-1)\left(1-z^{2}\right)^{N / 2}-N z(z-1)^{2}\left(1-z^{2}\right)^{N / 2-1}\right]
$$

One can explicitly check that this equation is satisfied by the perturbative expression of Equation (45) and $z(r)$ of Equation (38).

The longitudinal electric field inside the string is given by

$$
F^{03}=-4 \Lambda e^{-\Lambda r^{2}}\left[1+\mu\left(2 e^{-\Lambda r^{2}}\left(1-e^{-\Lambda r^{2}}\right)\right)^{N / 2} \cos N \theta\right]
$$

As before, the electric field is concentrated within the radius $\Lambda^{1 / 2}$ in the transverse plane, with an angular modulation of the transverse profile due to the additional $Z_{N}$ invariant potential.

\subsection{General Solution}

We now demonstrate the string solution beyond the simple perturbative approximation discussed above. Minimizing the energy functional Equation (42) yields the equations:

$$
\begin{aligned}
\frac{1}{e^{2}} \epsilon_{i j} \partial_{j} \chi \partial_{i} F & =\frac{\partial U}{\partial z} \\
\frac{1}{e^{2}} \epsilon_{i j} \partial_{j} z \partial_{i} F & =-\frac{\partial U}{\partial \chi}
\end{aligned}
$$

with

$$
F \equiv \frac{1}{2} F^{03}=\epsilon_{i j} \partial_{i} z \partial_{j} \chi,
$$

where $U$ is the potential of Equation (42).

These equations can be combined into:

$$
\frac{1}{2 e^{2}} \partial_{k}\left(F^{2}\right)=\partial_{k} U
$$

Requiring that the electric field vanishes at transverse infinity, as should be the case for any finite energy density configuration, we find

$$
F^{2}=2 e^{2} U ; \quad F=\sqrt{2 e^{2} U}
$$

Let us work in the modified polar coordinates $\left(\tau=r^{2}, \theta\right)$. We then have

$$
\partial_{\tau} z \partial_{\theta} \chi-\partial_{\theta} z \partial_{\tau} \chi=\sqrt{\frac{1}{2} e^{2} U}
$$

This equation has infinite number of solutions. This infinite degeneracy results from an unusual symmetry of the energy functional Equation (42). Consider the group of area-preserving diffeomorphisms on a plane $\operatorname{SDiff}\left(R^{2}\right)$

$$
(z(x), \chi(x)) \rightarrow\left(z\left(x^{\prime}\right), \chi\left(x^{\prime}\right)\right) ; \quad \frac{\partial\left(x^{\prime 1}, x^{\prime 2}\right)}{\partial\left(x^{1}, x^{2}\right)}=1
$$

These transformations leave the energy functional Equation (42) invariant. Therefore, any string solution can be transformed by a transformation Equation (53), generating an infinite number of degenerate solutions. Note that the longitudinal electric field is itself invariant under Equation (53), and thus, all the degenerate solutions have identical electric field and energy density profiles.

Interestingly, the transformations $\operatorname{SDiff}\left(R^{2}\right)$ of Equation (53) are diffeomorphisms on the coordinate space rather than on the field space. Thus, this is a different diffeomorphisms than $\operatorname{Sdiff}\left(S^{2}\right)$, which we discussed in the previous section and is explicitly broken by 
the potential $U$. The symmetry $\operatorname{SDiff}\left(R^{2}\right)$ is in a sense accidental since it only exists for configurations translationally invariant in one direction.

Let us now discuss two solutions related by $\operatorname{SDiff}\left(R^{2}\right)$. We can utilize the large symmetry by prescribing a simple dependence of $\chi$ on the angle $: \chi=\theta$. The equation for $z$ then follows from Equation (52).

$$
\partial_{\tau} z=\sqrt{\frac{1}{2} e^{2} U}=\sqrt{\Lambda^{2}(z-1)^{2}\left[1-2 \mu\left(1-z^{2}\right)^{N / 2} \cos N \theta\right]}
$$

The coordinate $\theta$ enters here as a parameter, and for a given value of $\theta$, the solution is

$$
\tau=\int_{-1}^{z(\tau)} d z \frac{1}{\sqrt{\Lambda^{2}(z-1)^{2}\left[1-2 \mu\left(1-z^{2}\right)^{N / 2} \cos N \theta\right]}}
$$

This has correct large distance asymptotic behavior since as $\tau \rightarrow \infty$, the function $z$ has to approach unity for the right hand side to diverge. It is easy to find the large distance asymptotics of the solution. When $z$ is close to unity, the term proportional to $\mu$ in the denominator can be neglected, and we have

$$
\tau=\int_{-1}^{z(\tau)} d z \frac{1}{\sqrt{\Lambda^{2}(z-1)^{2}}}
$$

which is solved by

$$
z(\tau \rightarrow \infty)=1-2 e^{-\Lambda \tau}
$$

This is identical to Equation (38), and thus, the IR asymptotics of the solution is not sensitive to the $Z_{N}$ perturbation.

As an example of another solution, we assume that $z$ has no angular dependence. We then have:

$$
\partial_{\tau} z \partial_{\theta} \chi=\sqrt{\Lambda^{2}(z-1)^{2}\left[1-2 \mu\left(1-z^{2}\right)^{N / 2} \cos N \chi\right]}
$$

This determines $\theta$ as a function of $r$ :

$$
\theta=\int_{0}^{\chi(r, \theta)} \frac{z^{\prime} d \chi}{\sqrt{\Lambda^{2}(z-1)^{2}\left[1-2 \mu\left(1-z^{2}\right)^{N / 2} \cos N \chi\right]}}
$$

The explicit solution is

$$
\theta=\frac{2}{N} \frac{z^{\prime}}{\sqrt{\Lambda^{2}(z-1)^{2}\left(1-2 \mu\left(1-z^{2}\right)^{N / 2}\right)}} F\left(\frac{N \chi}{2}, \frac{4 \mu\left(1-z^{2}\right)^{N / 2}}{2 \mu\left(1-z^{2}\right)^{N / 2}-1}\right)
$$

where $F(\phi, m)$ is the incomplete elliptic integral of the first kind:

$$
F(\phi, m)=\int_{0}^{\phi}\left(1-m \sin \theta^{2}\right)^{-1 / 2} d \theta
$$

Imposing on the solution the boundary condition

$$
\chi(\theta+2 \pi)=\chi(\theta)+2 \pi
$$

and using $F\left(\frac{k \pi}{2}, m\right)=k K(m)$, with $K(m)$-the complete elliptic integral of the first kind, gives

$$
2 \pi=\frac{4 z^{\prime}}{\sqrt{\Lambda^{2}(z-1)^{2}\left(1-2 \mu\left(1-z^{2}\right)^{N / 2}\right)}} K\left(\frac{4 \mu\left(1-z^{2}\right)^{N / 2}}{2 \mu\left(1-z^{2}\right)^{N / 2}-1}\right)
$$


It is easy to check that for $z \rightarrow 1$, this reduces to

$$
z^{\prime}=\Lambda(1-z)
$$

and thus, the IR asymptotics again is the same as in Equation (38).

\section{Discussion of the Model Mark 1}

In constructing our model, we have tried to follow the guide of $2+1$ dimensional gauge theories and, based on several requirements, "guess" a theory of scalar fields that may emulate the effective theory of $3+1$ dimensional gauge theories. The model we were led to is not quite satisfactory, but it does have several interesting and intriguing features.

First off, already in the Abelian limit, it is quite peculiar. It possesses an infinite dimensional global symmetry group, which is spontaneously broken by classical solutions of lowest energy. On the other hand, the observables that we would like to identify with physical quantities in QED turn out to be invariant under this symmetry. This may seem problematic; however, we note that a somewhat similar situation occurs in $2+1$ dimensions and, in general, in dual type descriptions. In $2+1$ dimensional gauge theories, the electromagnetic field is invariant under the action of the magnetic $U(1)$ symmetry, which does act nontrivially on the magnetic vortex field-the basic degree of freedom in the effective/'dual" description. In the present $3+1$ dimensional model, likewise, the electromagnetic field does not feel the action of the (infinite) global symmetry group Sdiff $\left(S^{2}\right)$, which does act nontrivially on the "fundamental" scalar fields of the effective theory.

The global $\operatorname{Sdiff}\left(S^{2}\right)$ symmetry is classically broken by the lowest energy configurations. This is similar to $2+1$ dimensions, but the situation is more involved. In $2+1$ dimensions, we had to deal with a standard symmetry breaking pattern of symmetry with a finite number of generators. In our $3+1$ dimensional model, on the other hand, the symmetry group is infinitely dimensional, and thus, the space of vacuum configurations is very large. It includes not only translationally invariant field configurations but also configurations with nontrivial spatial dependence. These configurations break translational invariance in addition to the global $\operatorname{Sdiff}\left(S^{2}\right)$ symmetry. This is not a unique situation, and in fact, such a situation is ubiquitous in condensed matter systems, but in relativistic field theories, it is quite rare. As a result, since the vacuum set has large entropy, it could well be that classical analysis fails in this model quite badly. Many of the classical vacua differ from each other only in the finite region of space. Generically in cases like this, upon quantization, these configurations become connected by tunneling transitions of finite probability. One, therefore, may expect that the quantum portrait of moduli space is very different from the classical one. This question is worth investigating on its own, but this goes far beyond the scope of the present work.

With a symmetry breaking perturbation, our model exhibits a simple classical mechanism of confinement of topological defects, such as in $2+1$ dimensions. However, some peculiarities are present again. We have discovered that string solutions are infinitely degenerate. The static energy of configurations translationally invariant in one direction has an additional diffeomorphism invariance. This is not the same invariance as in the Abelian limit, as the diffeomorphisms in question are transformations in coordinate space and not in the field space. Nonetheless, this symmetry leads to degeneracy between an infinite number of solutions, all of which have the same electric field. As far as the electric field profile is concerned, the solution, as far as we can ascertain, is unique. This infinite degeneracy makes one wonder about the fate of such strings in a quantum theory, given that they carry large entropy associated with the degeneracy.

All the peculiar features of the model stem from the nonconventional, higher derivative kinetic term required to have finite energy of a soliton in the absence of the potential. One could add the standard two derivative kinetic term $\partial^{\mu} \phi^{a} \partial_{\mu} \phi^{a}$ as a perturbation. Although we have not explored this possibility in detail, it is clear that this would lift the degeneracy between the different string solutions. With this additional kinetic term, our model becomes identical with the model proposed by Faddeev and Niemi in [7-11] as an 
effective theory of QCD. Note, however, that our proposed picture of confinement is very different from and in a way complementary to that of [7-11]. The authors of [7-11] are mostly interested in closed string solutions meant to represent the glueballs, while in our way of thinking, it is the open strings, with the endpoints representing "constituent gluons" that play the main role in analogy with $2+1$ dimensions $[2,3,16]$. In the Faddeev-Niemi model, stability of closed string solutions is ensured by nontrivial twisting of the phase of the scalar field along the string. Open strings, on the other hand, are not associated with twist and in principle can break into shorter strings, which is the case in QCD. The stability of classical strings solutions in a quantum theory is not absolute but is rather an approximate feature that arises dynamically provided the string endpoints are sufficiently heavy [17]. This endpoint mass suppresses quantum tunneling, which is responsible for the decay of long strings.

Finally, it is worthwhile noting that the addition of the two derivative kinetic term makes our model similar to the $C P^{1}$ model, which has been recently discussed in the literature in relation to effective models of confinement [18].

The large global symmetry of our model in the Abelian, which has no obvious parallel in QED, is worrisome. One can wonder if it is responsible at least partially for the absence of an arbitrary "two-photon state", as we have found here. It is, therefore, natural to try and eliminate this symmetry from the model. In the next section, we describe an approach to doing so by "gauging" this global symmetry. This amounts to restricting the Hilbert space of the model to states that are invariant under $\operatorname{Sdiff}\left(S^{2}\right)$.

\section{Gauging $\operatorname{Sdiff}\left(S^{2}\right)$}

In this section, we show how the global $\operatorname{Sdiff}(2)$ symmetry can be eliminated from the theory. The standard way of going about such a task is to "gauge" the symmetry, i.e., to impose the vanishment of the appropriate charge. It is usually employed to eliminate local symmetries; however, as a matter of principle, it can also be done for global symmetries. We will now describe this procedure.

Recall that the symmetry in question is

$$
z \rightarrow z+\frac{\partial G(z, \chi)}{\partial \chi}, \chi \rightarrow \chi-\frac{\partial G}{\partial z}
$$

with $G$ being an arbitrary function of the two variables $z$ and $\chi$ but does not explicitly depend on space-time coordinates.

This symmetry is associated with the conserved currents

$$
J_{v}^{G}=G(z, \chi) J_{v}=G(z, \chi) \partial^{\mu}\left(\partial_{\mu} z \partial_{\nu} \chi-\partial_{\mu} \chi \partial_{\nu} z\right) .
$$

where

$$
J_{v}=\partial^{\mu}\left(\partial_{\mu} z \partial_{\nu} \chi-\partial_{\mu} \chi \partial_{\nu} z\right)
$$

The corresponding charges are

$$
Q^{G}=\int d^{3} x G(z, \chi) J_{0}=\int d^{3} x G(z, \chi) \partial^{\mu}\left(\partial_{\mu} z \partial_{0} \chi-\partial_{\mu} \chi \partial_{0} z\right)
$$

We note for future use that the symmetry transformation can be written as a canonical transformation on a phase space spanned by $z$ and $\chi$.

$$
\delta z=\{z, G\} ; \quad \delta \chi=\{\chi, G\} ; \quad\{A, B\} \equiv \frac{\partial A}{\partial z} \frac{\partial B}{\partial \chi}-\frac{\partial A}{\partial \chi} \frac{\partial B}{\partial z}
$$

To gauge this symmetry, we first introduce the analog of the zeroth component of vector potential $\Lambda(z, \chi, t)$. Note that $\Lambda$ is not an arbitrary function of space-time coordinates but only a function of the field variables $z$ and $\chi$ and time $t$. 
We now change our definition of the "magnetic field" to

$$
B_{k}=2\left(\partial_{k} \chi \partial_{0} z-\partial_{0} \chi \partial_{k} z\right)-\partial_{k} \Lambda=2\left(\partial_{k} \chi \partial_{0} z-\partial_{0} \chi \partial_{k} z\right)-\frac{\partial \Lambda}{\partial z} \partial_{k} z-\frac{\partial \Lambda}{\partial \chi} \partial_{k} \chi
$$

Defining "covariant derivative" as

$$
\begin{aligned}
& \nabla_{0} \chi=\partial_{0} \chi+\frac{1}{2} \frac{\partial \Lambda}{\partial z} \\
& \nabla_{0} z=\partial_{0} z-\frac{1}{2} \frac{\partial \Lambda}{\partial \chi}
\end{aligned}
$$

we can write this as

$$
B_{k}=2\left(\partial_{k} \chi \nabla_{0} z-\nabla_{0} \chi \partial_{k} z\right)
$$

Note that this definition of covariant derivative implies for any function of $z$ and $\chi$

$$
\nabla_{0} \Phi(z, \chi)=\frac{d}{d t} \Phi-\frac{1}{2}\{\Phi, \Lambda\}
$$

With this altered definition of the magnetic field, and the electric field is still defined as

$$
E_{i}=2 \epsilon_{i j k} \partial_{j} z \partial_{k} \chi
$$

we now write the Lagrangian

$$
\mathcal{L}=-\frac{1}{2}\left(\vec{E}^{2}-\vec{B}^{2}\right)
$$

As we show now, this Lagrangian is gauge invariant. First, let us consider time independent transformations from Equation (69). Under this transformation, we define the transformation of $\Lambda$ as

$$
\delta \Lambda=-\{\Lambda, G\}=-\left[\frac{\partial \Lambda}{\partial z} \frac{\partial G}{\partial \chi}-\frac{\partial \Lambda}{\partial \chi} \frac{\partial G}{\partial z}\right]
$$

Note, that this equation should be understood as the change in the functional form of $\Lambda$ as a function of $z$ and $\chi$. With this definition and taking into account that the values of $z$ and $\chi$ change according to Equation (69), we find

$$
\Lambda^{\prime}\left(z^{\prime}, \chi^{\prime}\right)=\Lambda(z, \chi)
$$

Thus, it is easy to see that both $E_{i}$ and $B_{i}$ are invariant under the time-independent transformations Equations (69) and (76).

Now, consider time-dependent transformations, $G(z, \chi, t)$. The electric field is invariant under the time-dependent transformations as well. For the magnetic field, a straightforward calculation gives

$$
\begin{aligned}
2\left(\partial_{k} \chi \partial_{0} z-\partial_{0} \chi \partial_{k} z\right) & \rightarrow 2\left[\partial_{k}\left(\chi-\frac{\partial G}{\partial z}\right) \partial_{0}\left(z+\frac{\partial G}{\partial \chi}\right)-\partial_{0}\left(\chi-\frac{\partial G}{\partial z}\right) \partial_{k}\left(z+\frac{\partial G}{\partial \chi}\right)\right] \\
& =2\left(\partial_{k} \chi \partial_{0} z-\partial_{0} \chi \partial_{k} z\right)+2 \partial_{i} \partial_{0} G+O\left(G^{2}\right)
\end{aligned}
$$

Thus, if we define the transformation of $\Lambda$ as

$$
\delta \Lambda=2 \partial_{0} G-\{\Lambda, G\}
$$

we find that the magnetic field in Equation (72) is invariant.

To summarize, we have now constructed the Lagrangian, which is invariant under arbitrary time-dependent $\operatorname{Sdiff}\left(S^{2}\right)$ transformations. Physically, this gauge invariance means that the $\operatorname{Sidf} f\left(S^{2}\right)$ charges are required to vanish on physical configurations. Indeed, we can see that the equation of motion for $\Lambda$ is indeed equivalent to this constraint. We note 
that variation with respect to $\Lambda$ should be done with care since $\Lambda$ is not an independent field. One cannot vary space-time dependence of $\Lambda$ arbitrarily; instead, one has to vary the functional form of the dependence on the field $z$ and $\chi$.

Let us derive equations of motion for Lagrangian Equation (75). Varying with respect to $z$ and $\chi$, we obtain

$$
\begin{aligned}
& \partial_{i} \chi\left[\partial_{0} B_{i}+\epsilon_{i j k} \partial_{j} E_{k}\right]-\partial_{i} B_{i} \nabla_{0} \chi=0 \\
& \partial_{i} z\left[\partial_{0} B_{i}+\epsilon_{i j k} \partial_{j} E_{k}\right]-\partial_{i} B_{i} \nabla_{0} z=0
\end{aligned}
$$

or in relativistic notation

$$
\nabla^{v} \chi \partial^{\mu} \tilde{F}_{\mu v}=0 ; \quad \nabla^{v} z \partial^{\mu} \tilde{F}_{\mu v}=0
$$

These can be combined into a covariant conservation equation

$$
\nabla^{\mu} J_{\mu}^{G}=0
$$

with the current

$$
J_{\mu}^{G} \equiv G(z, \chi) \partial^{v} \tilde{F}_{\nu \mu}=0
$$

with arbitrary function $G$.

In addition, there is an equation obtained by differentiation with respect to $\Lambda$. To understand how to derive this equation, we can expand $\Lambda(z, \chi)$ in a complete basis of functions on a two-dimensional space, for example, by writing $\Lambda(z, \chi)=\int d p d q e^{i p z+i q \chi} \tilde{\Lambda}(p, q)$ and substituting it into the action, then differentiate with respect to $\tilde{\Lambda}$. The resulting equations are

$$
\int d^{3} x J_{0}^{G}=\int d^{3} x G(z, \chi) \partial_{i} B_{i}=0
$$

This equations are rather interesting. They put a large number of constraints on the divergence of the magnetic field. Unfortunately, the number of constraints is not large enough to ensure that magnetic monopole charge vanishes, as $G$ is only a function of two variables (at any given time), while the coordinate space is obviously three-dimensional.

One could ask whether the modification we made can help us find arbitrary two gluon states in the spectrum. Unfortunately, the answer is negative. The simplest way to see it is to realize that one can gauge fix the "vector potential" $\Lambda$ to zero-the Hamiltonian gauge of sorts. In this gauge, the dynamical equations of the model are identical with the equations of Mark 1. Thus, we do not have new solutions to the equations of motion. The gauging does eliminate those solutions that do not satisfy the constraint Equation (84), but it does not generate any new solutions to the equations of motion.

Thus, although it feels like gauging $\operatorname{Sdiff}\left(S^{2}\right)$ may be a step in the right direction, it is not sufficient. In the next section, we discuss a further modification of the model-Mark 2, which starts from the same premise but successfully reproduces the theory of free photon.

\section{The Model Mark 2}

The model of [5], despite having some interesting properties, fails to describe adequately the low energy dynamics of the Abelian limit. As we have learned from the previous section, gauging the $\operatorname{Sdiff}\left(S^{2}\right)$ symmetry does not solve the main problems of [5], i.e., on one hand, the constraints it imposes are not sufficient to ensure vanishing of magnetic charge density, and on the other hand, it does not allow for additional solutions of equations of motion that can be identified with multiphoton states of arbitrary polarization. Both of these deficiencies are associated with the fact that the "vector potential" $\Lambda$ is not a bona fide local degree of freedom but only a function of two variables $z$ and $\chi$. Let us extend our approach by allowing $\Lambda$ to become an independent function of space time. We, therefore, change our definition of magnetic field to [6]

$$
F^{\mu v}=\epsilon^{\mu \nu \alpha \beta}\left[\epsilon^{a b c} \phi_{a} \partial_{\alpha} \phi_{b} \partial_{\beta} \phi_{c}+(n \cdot \partial) n_{\alpha} \partial_{\beta} \Phi\right]
$$


Here, $n=(1,0,0,0)$ is a time-like vector of unit length and $\Phi$ is a scalar field [19]. This is a generalization of Equation (70) with $\Lambda \rightarrow \partial_{0} \Phi$.

We stress that, as opposed to the discussion in the previous section, $\Phi(x)$ is now a bona fide field that has a general dependence on space-time coordinates.

The Lagrangian, as before, is

$$
\mathcal{L}=\frac{1}{4} F_{\mu v} F^{\mu \nu}=-\frac{1}{2}\left(\vec{E}^{2}-\vec{B}^{2}\right)
$$

One may worry that since $n$ is chosen to be a time-like vector, the model is not a Lorentz invariant. Nevertheless, we will show below that the model possesses a Lorentz invariant super selection sector, and it is this sector that will turn out to be equivalent to QED.

We now have to understand what effect the modification has on the Abelian limit of the model. We will analyze its canonical structure and will demonstrate that it is identical to that of free electrodynamics. This applies to the commutators between the "electric" and "magnetic" fields and the Hamiltonian. We, thereby, demonstrate that the model is equivalent to the theory of a free noninteracting photon, even though it is not formulated in terms of a covariant vector potential field. We also derive the Lorentz transformation properties on the degrees of freedom of the model. We demonstrate that the fields $\phi^{i}$ are not covariant scalar fields but instead transform nontrivially and noncovariantly under the Lorentz group. We confirm that due to these modified transformation properties, the model retains Lorentz invariance.

\section{Equations of Motion and Canonical Structure}

\subsection{Equations of Motion}

As before, we use the following parametrization of the basic fields $\chi$ and $z$ via $\phi_{3}=z$ and $\phi_{1}+i \phi_{2}=\sqrt{1-z^{2}} e^{i \chi}$. The electromagnetic field can now be written as:

$$
F^{\mu v}=\epsilon^{\mu v \alpha \beta}\left[-2 \partial_{\beta} \chi \partial_{\alpha} z+n_{\alpha} \partial_{\beta} \partial_{0} \Phi\right]
$$

or explicitly

$$
\begin{gathered}
E_{i}=2 \epsilon_{i j k} \partial_{j} z \partial_{k} \chi \\
B_{k}=\left[2\left(\partial_{k} \chi \partial_{0} z-\partial_{0} \chi \partial_{k} z\right)-\partial_{k} \partial_{0} \Phi\right]
\end{gathered}
$$

Varying the action, Equation (86) yields the following equations of motion

$$
\begin{gathered}
\partial_{0} \partial_{k}\left[F_{i j} \epsilon^{i j 0 k}\right]=0=\partial_{0} \partial_{k} B_{k} \\
\partial_{\beta} \chi \partial_{\alpha}\left(F_{\mu \nu} \epsilon^{\mu \nu \alpha \beta}\right)=0=\partial_{k} \chi \partial_{\alpha}\left(F_{\mu \nu} \epsilon^{\mu \nu \alpha k}\right)=\partial_{k} \chi\left(\partial_{0} B_{k}+(\partial \times E)_{k}\right) \\
\partial_{\beta} z \partial_{\alpha}\left(F_{\mu \nu} \epsilon^{\mu \nu \alpha \beta}\right)=0=\partial_{k} z \partial_{\alpha}\left(F_{\mu \nu} \epsilon^{\mu \nu \alpha k}\right)=\partial_{k} z\left(\partial_{0} B_{k}+(\partial \times E)_{k}\right)
\end{gathered}
$$

The main difference with our previous attempt is Equation (90). This equation now means that the "magnetic charge density" $\partial_{k} B_{k}$ is locally conserved. In the current model, therefore, the magnetic charge density is time independent at any space point. Equation (90) imposes the existence of "super selection sectors" characterized by the value of the magnetic charge density at all spatial coordinates. Clearly, most of these sectors are not translationally invariant. In order to preserve translational invariance, we limit our consideration to the trivial sector with $\partial_{k} B_{k}=0$. The rest of our discussion pertains exclusively to this super selection sector.

Using this constraint on the magnetic field, we can invert Equations (91) and (92). (One has to be careful since there is an ambiguity in the inversion of Equations (91) and (92). In general, we find $\partial_{0} B_{k}+(\partial \times E)_{k}=\alpha E_{k}$, where $\alpha$ is an arbitrary constant. Nonetheless, 
since $B$ and $\partial \times E$ are pseudovectors, while $E$ is a vector, a nonvanishing value of $\alpha$ would violate parity. Imposing parity invariance on the solution sets $\alpha=0$.)

$$
\partial_{0} B_{k}+(\partial \times E)_{k}=0
$$

Recall that with the field strength components given by Equation (87), the "electric" equation

$$
\partial_{\mu} F^{\mu v}=0
$$

is satisfied identically. Thus, the equations of motion of the model Mark 2 are the full set of Maxwell's equations.

\subsection{The Hamiltonian}

Let us now turn to the Hamiltonian description of the model. The canonical momenta as calculated from Equation (87) are given by :

$$
\begin{array}{r}
p_{z}=\frac{\delta L}{\delta \partial_{0} z}=F_{i j} \epsilon^{i j 0 k} \partial_{k} \chi=2 B_{k} \partial_{k} \chi=2 \partial_{k} \chi\left[2\left(\partial_{k} \chi \partial_{0} z-\partial_{0} \chi \partial_{k} z\right)-\partial_{k} \partial_{0} \Phi\right] \\
p_{\chi}=\frac{\delta L}{\delta \partial_{0} \chi}=F_{i j} \epsilon^{i j k 0} \partial_{k} z=-2 B_{k} \partial_{k} z=-2 \partial_{k} z\left[2\left(\partial_{k} \chi \partial_{0} z-\partial_{0} \chi \partial_{k} z\right)-\partial_{k} \partial_{0} \Phi\right] \\
p_{\Phi}=\frac{\delta L}{\delta \partial_{0} \Phi}=\frac{1}{2} \partial_{k}\left(F_{i j} \epsilon^{i j 0 k}\right)=\partial_{k} B_{k}=\partial_{k}\left[2\left(\partial_{k} \chi \partial_{0} z-\partial_{0} \chi \partial_{k} z\right)-\partial_{k} \partial_{0} \Phi\right]
\end{array}
$$

The time derivatives of the fields can be expressed as:

$$
\begin{aligned}
& \dot{\chi}=\frac{1}{E^{2}}\left[p_{z}(z \chi)+p_{\chi} \chi^{2}+\epsilon_{i j k} \dot{\Phi}_{i} E_{j} \chi_{k}\right] \\
& \dot{z}=\frac{1}{E^{2}}\left[p_{z} z^{2}+p_{\chi}(z \chi)+\epsilon_{i j k} \dot{\Phi}_{i} E_{j} z_{k}\right]
\end{aligned}
$$

Using these equations, we express $p_{\Phi}$ as:

$$
p_{\Phi}=\partial_{k}\left[\frac{1}{E^{2}} \epsilon_{k l m} E_{l}\left(p_{z} z_{m}+p_{\chi} \chi_{m}\right)-\frac{1}{E^{2}} E_{k} E_{i} \dot{\Phi}_{i}\right]
$$

or in terms of a "vector potential"

$$
D_{k}=\frac{1}{E^{2}} \epsilon_{k l m} E_{l}\left(p_{z} z_{m}+p_{\chi} \chi_{m}\right)
$$

as

$$
p_{\Phi}=\partial_{k}\left(D_{k}-\hat{E}_{k} \hat{E}_{i} \dot{\Phi}_{i}\right)
$$

The Hamiltonian is then calculated as:

$$
H=\int d^{3} x\left[p_{z} \dot{z}+p_{\chi} \dot{\chi}+p_{\Phi} \dot{\Phi}-L\right]=\int d^{3} x \frac{1}{2}\left(E^{2}+B^{2}\right)
$$

In arriving at this expression, we have neglected a boundary term $\int d^{3} x \partial_{k}\left(B_{k} \dot{\Phi}\right)$.

\subsection{Canonical Structure}

In order to prove the equivalence between Mark 2 and QED, we have to show that the canonical commutation relations of $E_{i}$ and $B_{i}$ are identical in the two theories. We do this in the present section.

Since all components of the electric field in Mark 21 are functions only of coordinates and not canonical momenta, they clearly commute with each other

$$
\left[E_{i}(x), E_{j}(y)\right]=0
$$


In order to calculate the commutation relations between electric and magnetic fields, we restrict ourselves explicitly to the zero magnetic charge density super selection sector and set $p_{\Phi}=0$. Equation (102) then becomes

$$
\frac{\partial_{k} D_{k}}{E}=\hat{E}_{k} \partial_{k}\left(\frac{\hat{E}_{i} \dot{\Phi}_{i}}{E}\right)
$$

where we have used $\partial_{k} E_{k}=0$.

Formally, the solution is written as

$$
\hat{E}_{i} \dot{\Phi}_{i}=E(x) \int_{-\infty}^{x} d l_{C} \frac{\partial_{k} D_{k}}{E}
$$

In this expression, the integration contour $C$ starts at $x$ and ends at some point at spatial infinity. The direction along the contour at every point is parallel to the direction of the electric field at this point.

Using the definition of $D$, we have:

$$
B_{k}=D_{k}-E_{k} \int_{-\infty}^{x} d l_{C} \frac{\partial_{m} D_{m}}{E}
$$

Consider first the following auxiliary quantity

$$
\begin{aligned}
{\left[E_{i}(x), D_{k}(y)\right] } & =2 i \frac{E_{l}(y)}{E^{2}(y)} \epsilon_{i a b} \epsilon_{k l m}\left[\partial_{a}^{x} \delta(x-y) \chi_{b}(x) z_{m}(y)+\partial_{b}^{x} \delta(x-y) z_{a}(x) \chi_{m}(y)\right] \\
& =2 i \frac{E_{l}(y)}{E^{2}(y)} \epsilon_{i a b} \epsilon_{k l m} \partial_{a}^{x} \delta(x-y)\left[\chi_{b}(y) z_{m}(y)-z_{b}(y) \chi_{m}(y)\right] \\
& =i \hat{E}_{l}(y) \hat{E}_{c}(y) \epsilon_{i a b} \epsilon_{k l m} \epsilon_{c m b} \partial_{a}^{x} \delta(x-y)=i\left[\epsilon_{i a k}-\hat{E}_{b}(y) \hat{E}_{k}(y) \epsilon_{i a b}\right] \partial_{a}^{x} \delta(x-y)
\end{aligned}
$$

Using this, we calculate

$$
\begin{aligned}
{\left[E_{i}(x), B_{k}(y)\right] } & =\left[E_{i}(x), D_{k}(y)\right]-E_{k}(y) \int_{-\infty}^{y} d l_{C} \frac{\partial_{m}^{t}\left[E_{i}(x), D_{m}(t)\right]}{E(t)} \\
& =\left[E_{i}(x), D_{k}(y)\right]-E_{k}(y) \int_{-\infty}^{y} d l_{C} \frac{1}{E(t)} \partial_{m}^{t}\left[\left(\epsilon_{i a m}-\hat{E}_{b}(t) \hat{E}_{m}(t) \epsilon_{i a b}\right) \partial_{a}^{x} \delta(x-t)\right] \\
& =\left[E_{i}(x), D_{k}(y)\right]+E_{k}(y) \int_{\infty}^{y} d l_{C} \hat{E}_{m}(t) \partial_{m}^{t}\left(\frac{\hat{E}_{b}(t)}{E(t)} \epsilon_{i a b} \partial_{a}^{x} \delta(x-t)\right) \\
& =i \epsilon_{i a k} \partial_{a}^{x} \delta(x-y)
\end{aligned}
$$

Here, it was important that the integration contour $C$ is parallel to the electric field everywhere. In addition, we have assumed that all the fields vanish at the spatial boundary.

The commutator Equation (109) is identical to the commutator of corresponding quantities in QED.

We now consider the commutator of the magnetic fields.

It is easy to see that $\left[B_{i}(x), B_{a}(y)\right]=0$ as long as the curve $C_{x}$ that enters the definition of $B_{i}(x)$ in Equation (107) does not pass through the point $y$, and $C_{y}$ does not pass through $x$. If this condition is not satisfied, direct evaluation of the commutator is not easy. Instead, we argue indirectly. One can straightforwardly obtain a number of relations involving the commutator of interest. Consider, for instance,

$$
\left[B_{i}(x) \partial_{i} \chi(x), B_{j}(y) \partial_{j} z(y)\right]=\left[p_{z}(x), p_{\chi}(y)\right]=0
$$

Trivially: 


$$
\begin{aligned}
& B_{i}(x) \partial_{j} z(y)\left[\partial_{i} \chi(x), B_{j}(y)\right]+B_{j}(y) \partial_{i} \chi(x)\left[B_{i}(x), \partial_{j} z(y)\right]+\partial_{i} \chi(x) \partial_{j} z(y)\left[B_{i}(x), B_{j}(y)\right]= \\
& \left(B_{i}(x) \partial_{j} z(y) \partial_{i}^{(x)} \frac{\partial D_{j}(y)}{\partial p_{\chi}(x)}-B_{j}(y) \partial_{i} \chi(x) \partial_{j}^{(y)} \frac{\partial D_{i}(x)}{\partial p_{z}(y)}\right)+\partial_{i} \chi(x) \partial_{j} z(y)\left[B_{i}(x), B_{j}(y)\right]= \\
& \left(B_{i}(y) \partial_{i}^{(x)} \delta(x-y)+B_{i}(x) \partial_{i}^{(y)} \delta(x-y)\right)+\partial_{i} \chi(x) \partial_{j} z(y)\left[B_{i}(x), B_{j}(y)\right]= \\
& \partial_{i} \chi(x) \partial_{j} z(y)\left[B_{i}(x), B_{j}(y)\right]=0
\end{aligned}
$$

Similarly,

$$
\partial_{i} z(x) \partial_{j} z(y)\left[B_{i}(x), B_{j}(y)\right]=\partial_{i} \chi(x) \partial_{j} \chi(y)\left[B_{i}(x), B_{j}(y)\right]=0
$$

Using $\partial_{k} B_{k}=0$, we obtain:

$$
\partial_{i} z(x) \partial_{j}^{y}\left[B_{i}(x), B_{j}(y)\right]=\partial_{i} \chi(x) \partial_{j}^{y}\left[B_{i}(x), B_{j}(y)\right]=\partial_{i}^{x} \partial_{j}^{y}\left[B_{i}(x), B_{j}(y)\right]=0
$$

Defining the matrix $M_{i j}(x, y) \equiv\left[B_{i}(x), B_{j}(y)\right]$, we, therefore, find that it is antisymmetric under the exchange $(i, x) \leftrightarrow(j, y)$ and satisfies the set of equations (Equations (111)(113)). The general solution for these constraints can be written as

$$
M_{i j}(x, y)=E_{i}(x) F_{j}(y)-E_{j}(y) F_{i}(x)
$$

with $F_{i}(x)$ being an arbitrary vector function. However, we already saw that when $x$ does not belong to $C_{y}$ and $y$ does not belong to $C_{x}$, then $M_{i j}(x, y)=0$. This determines $F_{i}(x)=0$, so that finally:

$$
\left[B_{i}(x), B_{j}(y)\right]=0
$$

for all $x, y$.

\section{Lorentz Transformations of the Fields}

We now wish to discuss the properties of the fields $z$ and $\chi$ under Lorentz transformations. The rotational transformation properties of $z$ and $\chi$ are clearly those of a scalar field. This is obvious since the rotational invariance is represented in our model in the standard linear manner. This is not the situation with Lorentz boosts. The electric and magnetic fields are components of a covariant Lorentz tensor, and therefore, it is clear that $z$ and $\chi$ cannot be covariant scalar fields.

We take the following parametrization of infinitesimal Lorentz transformations:

$$
\begin{aligned}
z(x) & \rightarrow z\left(\Lambda^{-1} x\right)=(1+\beta \Delta) z(x)+a \\
\chi(x) & \rightarrow \chi\left(\Lambda^{-1} x\right)=(1+\beta \Delta) \chi(x)+b \\
\Theta(x) \equiv \partial_{0} \Phi & \rightarrow \Theta\left(\Lambda^{-1} x\right)=\Theta(x)+c
\end{aligned}
$$

Here, $\beta$ is the boost parameter and $\Delta \equiv \omega^{\mu}{ }_{v} x^{v} \partial_{\mu}$ with $\omega_{\nu}^{\mu}$-an antisymmetric generator of Lorentz transformation. The boost in the direction of a unit vector $\hat{n}$ is generated by $\omega_{0}^{i}=\hat{n}_{i}$. The terms involving $a, b$, and $c$ are not canonical, and we will determine them requiring that $F^{\mu v}$ transforms as a covariant tensor.

Let us consider first a boost in the direction of the first axis, $\hat{n}=(1,0,0)$. The components of the field strength tensor transform as

$$
E_{2}(x) \rightarrow E_{2}\left(\Lambda^{-1} x\right)-\beta B_{3}\left(\Lambda^{-1} x\right)
$$

Writing this in terms of $z, \chi$, and $\Theta$ and using Equation (116), we have:

$$
E_{2}(x)=2\left[\partial_{3} z(x) \partial_{1} \chi(x)-\partial_{1} z(x) \partial_{3}(x)\right] \rightarrow 2\left[\partial_{3} z\left(\Lambda^{-1} x\right) \partial_{1} \chi\left(\Lambda^{-1} x\right)-\partial_{1} z\left(\Lambda^{-1} x\right) \partial_{3}\left(\Lambda^{-1} x\right)\right]
$$


Comparing the two we obtain:

$$
-\beta \partial_{3} \Theta+2\left[\partial_{3} z \partial_{1} b+\partial_{3} a \partial_{1} \chi-\partial_{1} z \partial_{3} b-\partial_{1} a \partial_{3} \chi\right]=0
$$

Similarly, the transformation of $E_{1}$ yields

$$
2\left(\partial_{2} z \partial_{3} b+\partial_{2} a \partial_{3} \chi-\partial_{3} z \partial_{2} b-\partial_{3} a \partial_{2} \chi\right)=0
$$

and that of $E_{3}$ :

$$
\beta \partial_{2} \Theta+2\left[\partial_{1} z \partial_{2} b+\partial_{1} a \partial_{2} \chi-\partial_{2} z \partial_{1} b-\partial_{2} a \partial_{1} \chi\right]=0
$$

Introducing $f_{i}=2\left(a \partial_{i} \chi-b \partial_{i} z\right)$, and $u_{i}=\left(0, \beta \partial_{3} \Theta,-\beta \partial_{2} \Theta\right)$, the above equations combine into

$$
\epsilon_{i j k} \partial_{j} f_{k}=u_{i}
$$

The general solution for $f$ is:

$$
\begin{aligned}
f_{i} & =-\frac{\epsilon_{i j k} \partial_{j} u_{k}}{\partial^{2}}+\partial_{i} \tilde{\lambda} \\
& =\beta \hat{n}_{i} \Theta+\partial_{i} \lambda
\end{aligned}
$$

where

$$
\tilde{\lambda}-\beta \frac{\hat{n}_{i} \partial_{i}}{\partial^{2}} \Theta=\lambda
$$

with $\lambda$ still to be determined.

Noting that Equations (123) and (89) become the same under the substitution, Equation (123) for $a$ and $b$ can be solved as

$$
\begin{aligned}
& \partial_{0} z \rightarrow a \\
& \partial_{0} \chi \rightarrow b \\
& \partial_{0} \Phi \rightarrow \lambda \\
& B_{k} \rightarrow \beta \Theta \hat{n}_{k}
\end{aligned}
$$

Using Equations (98) and (99), we find:

$$
\begin{aligned}
& a=\frac{1}{E^{2}}\left(\beta \Theta \hat{n}_{i}+\lambda_{i}\right) \epsilon_{i j k} E_{j} z_{k} \\
& b=\frac{1}{E^{2}}\left(\beta \Theta \hat{n}_{i}+\lambda_{i}\right) \epsilon_{i j k} E_{j} \chi_{k}
\end{aligned}
$$

Now, Equation (123) becomes an equation for $\lambda$ :

$$
E_{i}\left(\beta \Theta \hat{n}_{i}+\partial_{i} \lambda\right)=0
$$

which yields:

$$
\lambda(x)=-\beta \int_{\infty}^{x} d l_{C} \hat{E}_{i} \hat{n}_{i} \Theta
$$

where as before, the contour $C$ is locally parallel to the vector $E_{i}$.

The function $c$ can similarly be determined by considering the transformation of the magnetic field.

$$
B_{1}(x) \rightarrow B_{1}\left(\Lambda^{-1} x\right)=2\left[\partial_{1} \chi\left(\Lambda^{-1} x\right) \partial_{0}\left(\Lambda^{-1} x\right)-\partial_{0} \chi\left(\Lambda^{-1} x\right) \partial_{1} z\left(\Lambda^{-1} x\right)\right]-\partial_{1} \Theta\left(\Lambda^{-1} x\right)
$$

yields

$$
2\left[\partial_{1} \chi \partial_{0} a+\partial_{1} b \partial_{0} z-\partial_{0} \chi \partial_{1} a-\partial_{0} b \partial_{1} z\right]-\partial_{1} c+\beta \Delta \partial_{1} \Theta=0
$$


Similarly, the transformation of $B_{2}$ and $B_{3}$ gives

$$
\begin{aligned}
& 2\left[\partial_{2} \chi \partial_{0} a+\partial_{0} z \partial_{2} b-\partial_{0} \chi \partial_{2} a-\partial_{2} z \partial_{0} b\right]-\partial_{2} c+\beta \Delta \partial_{2} \Theta=0 \\
& 2\left[\partial_{3} \chi \partial_{0} a+\partial_{0} z \partial_{3} b-\partial_{0} \chi \partial_{3} b-\partial_{3} z \partial_{0} b\right]-\partial_{3} c+\beta \Delta \partial_{3} \Theta=0
\end{aligned}
$$

These can be combined into a single vector equation

$$
\partial_{0} f_{i}-\partial_{i} f_{0}-\partial_{i} c+\beta \Delta \partial_{i} \Theta=0
$$

Using Equation (123), we write this:

$$
\partial_{i}\left[\partial_{0} \lambda-f_{0}-c+\beta \Delta \Theta\right]=0
$$

yielding

$$
\begin{aligned}
c & =2\left(a \partial_{0} \chi-b \partial_{0} z\right)-\partial_{0} \lambda-\beta \Delta \Theta \\
& =\beta\left[\frac{2}{E^{2}} \epsilon_{i j k} E_{j}\left(\partial_{0} \chi z_{k}-\partial_{0} z \chi_{k}\right)\left[\Theta \hat{n}_{i}-\partial_{i} \int_{\infty}^{x} d l_{C} \hat{E}_{l} \hat{n}_{l} \Theta\right]+\partial_{0} \int_{\infty}^{x} d l_{C} \hat{E}_{i} \hat{n}_{i} \Theta-\Delta \Theta\right]
\end{aligned}
$$

To summarize, the fields $z, \chi$, and $\Phi$ under Lorentz boost transform according to Equation (116) with $a, b$, and $c$ given in Equations (126), (128), and (134). These somewhat complicated transformation properties ensure that electromagnetic fields are components of the covariant Lorentz tensor.

\section{Discussion of Model MARK 2}

Our amended model (Mark 2) is equivalent to the theory of a free photon. We were led to this model by our wish to eliminate the global $\operatorname{Sdiff}\left(S^{2}\right)$ symmetry but had to go further from the original model in order to achieve equivalence with QED. What is the fate of $\operatorname{Sdiff}\left(S^{2}\right)$ in Mark 2? It is indeed easy to see that this symmetry is gauged. In order to see that, let us write

$$
\partial_{0} \Phi=\Lambda(z, \chi, t)+\partial_{0} \bar{\Phi}
$$

Assigning to $\Lambda$ the same transformation properties under $\operatorname{Sdiff}\left(S^{2}\right)$ as before and requiring $\bar{\Phi}$ to be invariant, we see that the Lagrangian Equation (86) is indeed invariant under the $\operatorname{Sdiff}\left(S^{2}\right)$ global gauge transformation. Note that the decomposition Equation (135) is always possible, given that $\Phi$ is an arbitrary function of space-time coordinates. It is important that we have been able to obtain the theory of a free photon. Our main goal, however, was (and remains) to understand confinement in the Non-Abelian case. Here, the road is still very long and winding, and at this point, there are mainly questions. We need to generalize our model in several directions. First, charged states have not been included in the model. This should be relatively straightforward to mitigate. As suggested in [5], we should relax the constraint of constant length of the sigma model field $\phi^{a}$ and instead endow the modulus field $\phi^{2}$ with nontrivial dynamics. This will soften the classical behavior of the model in UV and will lead to UV finite energy of charged states. The configuration space of our model is $S O(3) \times R$, with the $S O(3)$ symmetry broken spontaneously to $O(2)$. The moduli space should, therefore, have a nontrivial homotopy group $\Pi_{2}(M)=Z$ and allow for a nontrivial topological charge, which is identified with the electric charge. (There may be some subtlety in this argument related to the fact that the global gauge group $\operatorname{Sdiff}\left(S^{2}\right)$ has to be modded out. However, since the gauge transformation is global, we do not anticipate any problems.)

The more complicated question is how to extend this model into the Non-Abelian regime. Following the logic of [5], we should add a perturbation thats explicitly breaks the global symmetry of the model and via this breaking generates a linear potential between the charges. Here, first of all, we need to understand whether this perturbation should preserve the $\operatorname{Sdiff}\left(S^{2}\right)$ gauge symmetry or should break it explicitly. Such a global gauge symmetry was not present in $2+1$ dimensional models [2,3], and we lack guidance on 
this question from $2+1 \mathrm{~d}$. It seems likely that the $\operatorname{Sdiff}\left(S^{2}\right)$ should be preserved by the perturbation. If that is the case, the type of perturbations considered in [5] do not fit the bill. Perhaps one should deal directly with the breaking of the generalized magnetic symmetry-the symmetry generated by the magnetic flux $[12,13]$ in terms of its order parameter-the t Hooft loop [1].

A rough idea of how this can work is the following. Let us try to define an operator that breaks the generalized magnetic symmetry. This should be an analog of a t Hooft loop operator, except it should have end points, so rather a t Hooft line operator. We write the following bilocal expression

$$
V(x, y)=\exp \left(i \int_{t=-\infty}^{t=0} d t A_{0}(x, t)\right) \exp \left(i \int_{x}^{y} A_{i}(z, t=0) d z^{i}\right) \exp \left(i \int_{t=0}^{t=-\infty} d t A_{0}(y, t)\right)
$$

Here, the components of dual vector potential are chosen as $A_{i}=z \partial_{i} \chi$ and $A_{0}=z \partial_{0} \chi+\frac{1}{2} \partial_{0} \Phi(x)$. Given the $\operatorname{Sdiff}\left(S^{2}\right)$ transformation properties of the various operators, we have

$$
\begin{gathered}
A_{i} \rightarrow A_{i}+\partial_{i}\left[G-z \frac{\partial G}{\partial z}\right] \\
A_{0} \rightarrow A_{0}+\frac{d}{d t}\left[G(z, \chi ; t)-z \frac{\partial G}{\partial z}\right]
\end{gathered}
$$

Under the assumption that the fields vanish at infinity, it is easy to see that the operator Equation (136) is invariant under $\operatorname{Sdiff} f\left(S^{2}\right)$. In terms of its quantum numbers, this operator essentially creates a monopole-antimonopole pair at points $x$ and $y$. For infinitesimally close points $y=x+\epsilon$, this becomes

$$
V\left(x, \epsilon_{i}\right)=1+i \epsilon_{i}\left[z \partial_{i} \chi-\partial_{i} \Phi-\partial_{i} \int_{-\infty}^{0} d t z \partial_{0} \chi\right]+\epsilon_{i} \epsilon_{j}\left[z \partial_{i} \chi-\frac{1}{2} \partial_{i} \Phi-\partial_{i} \int_{-\infty}^{0} d t z \partial_{0} \chi\right]\left[z \partial_{j} \chi-\frac{1}{2} \partial_{i} \Phi-\partial_{j} \int_{-\infty}^{0} d t z \partial_{0} \chi\right]
$$

We could contemplate averaging this operator over the direction of the point splitting vector $\epsilon$, which would kill the linear in $\epsilon$ term and would result in a term reminiscent of the gauge invariant Stueckelberg mass for the dual vector potential [20]. Adding an $n$-th power of such an operator as a perturbation to the Lagrangian would seem a reasonable way to proceed in order to break the generalized magnetic symmetry to the $Z_{N}$ subgroup.

Unfortunately, Equation (139) contains a term that is nonlocal in time. Thus, adding it to the Lagrangian would lead to nonlocal in time theory, which amounts to adding extra degrees of freedom in disguise. Although this may turn out to be necessary, it is clearly outside the rather tight framework that we have set out to ourselves from the beginning. Thus, before taking this route, a better understanding is necessary. We hope to be able to make progress in this approach in the future.

Author Contributions: Both authors contributed equally. Both All authors have read and agreed to the published version of the manuscript.

Funding: I.B.I. is partially supported by TUBITAK (The Scientific and Technological Research Council of Turkey) under the grant no 117F203. A.K. is supported by the NSF Nuclear Theory grant 1913890.

Institutional Review Board Statement: Not applicable.

Informed Consent Statement: Not applicable.

Data Availability Statement: Not applicable.

Conflicts of Interest: The authors declare no conflict of interest. 


\section{Appendix A}

In this appendix, we show that the model considered in this paper does not admit two-photon solutions with arbitrary polarizations. We are looking for two-photon solutions for which the electromagnetic tensor is of the form:

$$
\tilde{F}_{\mu \nu}=\partial_{[\mu} z \partial_{\nu]} \chi=A\left(k_{\mu} \epsilon_{v}^{1}-k_{\nu} \epsilon_{\mu}^{1}\right) \cos k x+B\left(p_{\mu} \epsilon_{v}^{2}-p_{\nu} \epsilon_{\mu}^{2}\right) \cos p x
$$

For simplicity, we choose the case when the first photon has momentum $k$ in $x$-direction and polarization $a$ in $y$-direction, while the second photon has momentum $p$ in $y$-direction and polarization $b$ in $z$-direction. Note that this case is not covered by our construction of two-photon states in the body of the paper.

Now, for components of $\tilde{F}_{\mu \nu}$, we have:

$$
\begin{gathered}
\partial_{[0} z \partial_{1]} \chi=0=\partial_{[1} z \partial_{3]} \chi=0 \\
\partial_{[0} z \partial_{2]} \chi=k a \cos k x=-\partial_{[1} z \partial_{2]} \chi \\
\partial_{[0} z \partial_{3]} \chi=p b \cos p x=-\partial_{[2} z \partial_{3]} \chi
\end{gathered}
$$

Introducing new coordinates $(x, y, z, t) \rightarrow(\bar{x}=t-x, \bar{y}=t-y, \bar{t}=t, \bar{z}=z)$ and using unbarred symbols for notational simplicity, we have:

$$
\begin{gathered}
\partial_{[t} z \partial_{y]} \chi=\partial_{[t} z \partial_{z]} \chi=\partial_{[x} z \partial_{z]} \chi=0 \\
\partial_{[t} z \partial_{x]} \chi=\partial_{[x} z \partial_{y]} \chi=-k a \cos k x \\
\partial_{[y} z \partial_{z]} \chi=p b \cos p y
\end{gathered}
$$

These equations have no solutions. Assuming $\partial_{t} z \neq 0$, the first two equations in Equation (A5) imply $\partial_{y} z \partial_{z} \chi-\partial_{z} z \partial_{y} \chi=0$, which contradicts Equation (A7). Alternatively, assuming $\partial_{t} z=0$, implies vanishing of either $\partial_{t} \chi$ or two other partial derivatives of $z$. It is then easy to see that both these options are in conflict with the rest of the equations. The result is that a two-photon state with this polarization pattern cannot be constructed in this model.

The model also contains solutions that do not satisfy the homogeneous Maxwell equation. As an example of such a solution consider the configuration

$$
\chi=\sin p \cdot x ; \quad z=\sin k \cdot x
$$

It is easy to see that this configuration satisfies equations of motion provided

$$
(p \cdot k)^{2}-p^{2} k^{2}=0
$$

A simple example is a light-like momentum $k^{\mu}$ and a space-like momentum $p^{\mu}$ satisfying $p \cdot k=0$. This yields the dual field strength

$$
\tilde{F}_{\mu v} \propto\left(k_{\mu} p_{v}-k_{\nu} p_{\mu}\right)[\cos (p+k) \cdot x+\cos (p-k) \cdot x]
$$

which is not conserved

$$
\partial^{\mu} \tilde{F}_{\mu \nu} \propto p^{2} k_{\nu}[\sin (p+k) \cdot x+\sin (p-k) \cdot x]
$$

In fact, both momenta $k+p$ and $k-p$ are space-like, and thus, $\tilde{F}_{\mu v}$ looks tachyonic. However, as mentioned in the Discussion, since the model classically has many degenerate vacua with broken translational invariance, the interpretation of classical solutions as excitations is not so clear. 


\section{References}

1. Hooft, G. On the Phase Transition Towards Permanent Quark Confinement. Nucl. Phys. B 1978, 138, 1-25. [CrossRef]

2. Kovner, A. At the Frontier of Particle Physics; Shifman, M., Ed.; World Scientific: Singapore, 2001; Volume 3, pp. 1777-1825;

3. IKogan, I.; Kovner, A. At the Frontier of Particle Physics; Shifman, N., Ed.; World Scientific: Singapore, 2002; Volume 4, pp. $2335-2407$.

4. Altes, C.K.; Kovner, A. Magnetic Z(N) symmetry in hot QCD and the spatial Wilson loop. Phys. Rev. D 2000, 62, 096008. [CrossRef]

5. Kovner, A.; Ilhan, I.B. Curious case of an effective theory. Phys. Rev. D 2013, 88, 125004.

6. Kovner, A.; Ilhan, I.B. Photons without vector fields. Phys. Rev. D 2016, 93, 025015.

7. Faddeev, L.D.; Niemi, A.J. Stable knot-like structures in classical field theory. Nature 1997, 387, 58-61. [CrossRef]

8. Faddeev, L.D.; Niemi, A.J. Partially Dual Variables in SU(2) Yang-Mills Theory. Phys. Rev. Lett. 1999, 82, 1624. [CrossRef]

9. Faddeev, L.D.; Niemi, A.J. Partial duality in SU(N) Yang-Mills theory. Phys. Lett. B 1999, 449, 214-218. [CrossRef]

10. Faddeev, L.D.; Niemi, A.J. Decomposing the Yang-Mills Field. Phys. Lett. B 1999, 464, 90-93. [CrossRef]

11. Faddeev, L.D.; Niemi, A.J. Spin-Charge Separation, Conformal Covariance and the SU(2) Yang-Mills Theory. Nucl. Phys. B 2007, 776, 38-65. [CrossRef]

12. Kovner, A.; Rosenstein, B. New Look at $Q E D_{4}$ : The Photon as a Goldstone Boson and the Topological Interpretation of Electric Charge. Phys. Rev. D 1994, 49, 5571-5581. [CrossRef]

13. Gaiotto, D.; Kapustin, A.; Seiberg, N.; Willett, B. Generalized Global Symmetries. JHEP 2015, 2, 172. [CrossRef]

14. Guendelman, E.I.; Nissimov, E.; Pacheva, S. Volume-Preserving Diffeomorphisms' versus Local Gauge Symmetry. Phys. Lett. B 1995, 360, 57-64. [CrossRef]

15. Takasaki, K. Turku 1991, Proceedings, Topological and Geometrical Methods in Field Theory; Mickelsson, J., Pekonen, O., Eds.; World Scientific: River Edge, NJ, USA, 1992; pp. 383-397.

16. Kovner, A.; Rosenstein, B. Strings and string breaking in 2+1 dimensional nonabelian theories. JHEP 1998, 9809, 003. [CrossRef]

17. Greensite, J. An introduction to the confinement problem. Lect. Notes Phys. 2011, 821, 1-211.

18. Gorsky, A.; Shifman, M.; Yung, A. Revisiting the Faddeev-Skyrme Model and Hopf Solitons. Phys. Rev. D 2013, 88, 045026. [CrossRef]

19. Gliozzi, F. String-like topological excitations of the electromagnetic field. Nucl. Phys. B 1978, 141, 379-390. [CrossRef]

20. Stueckelberg, E.C.G. Interaction energy in electrodynamics and in the field theory of nuclear forces. Helv. Phys. Acta 1938, 11, 225. 